\title{
Seguridad metropolitana mediante el uso coordinado de Drones
}

\section{Metropolitan security through the coordinated use of Drones}

\author{
Jairo Eduardo Márquez Díaz \\ Universidad de Cundinamarca extensión Chía \\ jairo.marquez@ucundinamarca.edu.co
}

(Tipo de Artículo: Investigación científica y tecnológica. Recibido: 04/11/2017. Aprobado: 04/12/2017)

\begin{abstract}
Resumen. En el presente artículo se expone el estudio de factibilidad acerca de la tecnología existente en drones y tecnologías conexas, con miras a establecer su potencial aplicación en una vasta red interconectada inteligente, trabajando al unísono en un entorno metropolitano, con miras a actuar como sistema de ayuda a las fuerzas del orden y de rescate en sus labores, por medio del escaneo, monitoreo y seguimiento de eventos in situ que afectan la seguridad y el orden público. Para ello, se realiza una exposición acerca de la integración de tecnologías emergentes estándar y avanzadas disponibles en el mercado, que son un factor fundamental para el desarrollo del proyecto, el cual tiene símiles en algunos contextos a nivel mundial, donde tecnologías tales como la robótica, la computación móvil, la sensórica, el internet de las cosas, la inteligencia artificial y los sistemas de comunicación, entre otros, van aportar lo suyo en este tipo de propuesta.
\end{abstract}

Abstract. This article presents the feasibility study about the existing technology in drones and related technologies, with a view to establishing its potential application in a vast interconnected intelligent network, working in unison in a metropolitan environment, with a view to acting as a system of helps the law enforcement and rescue forces in their work, through the scanning, monitoring and follow-up of on-site events that affect security and public order. For this, an exhibition is made about the integration of standard and advanced emerging technologies available in the market, which are a fundamental factor for the development of the project, which has similes in some contexts worldwide, where technologies such as robotics, mobile computing, the sensory, the internet of things, artificial intelligence and communication systems, among others, will contribute their own in this type of proposal.

Palabras clave. Autonomía, Drones, Energía híbrida, Inteligencia artificial, Monitoreo, Seguridad metropolitana, Sensórica, sistema de comunicación.

Keywords. Artificial intelligence, Autonomy, communication system, Drones, Hybrid energy, Monitoring, Metropolitan security, sensorics.

DOI 10.21500/20275846.3299

\section{Introducción}

Lo que se expone a continuación se basa en tecnologías existentes y en aquellas que están en vías de desarrollo 0 en proceso de implementación, enfocados a dar solución a una problemática global sobre seguridad en las grandes ciudades. Bajo esta premisa, se presentó una propuesta de trabajo final al curso TechniCity desarrollado para la Universidad Estatal de Ohio en el año 2016, la cual avaló a través de sus expertos, en la que se estableció su viabilidad y potencial aplicación a un futuro cercano, en particular la forma de recarga de una flota de drones, el aprovechamiento de la energía híbrida en un entorno metropolitano, la comunicación y monitoreo, entre otros. En este sentido, se presenta el trabajo bajo un modelo actualizado, conceptualizado y contextualizado a la comunidad científica y público en general, exhortando a incursionar en proyectos equivalentes y/o similares, con miras a mejorar la calidad de vida de las personas, el medioambiente y sociedad en general.

En la actualidad el principal uso de los drones de altas prestaciones está enfocado al campo militar, en particular misiones de reconocimiento y monitoreo, y en algunos casos de ataque. A nivel civil, el uso de los drones ha incursionado en varios nichos del mercado tales como: seguridad (detección y seguimiento), control fronterizo, búsqueda y rescate de personas, asistencia médica, cartografía aérea, control y prevención de incendios forestales, vigilancia, exploración, geología, agricultura de precisión, pesca, construcción e inspecciones, monitoreo del medio ambiente, movilidad y control de tráfico, publicidad, eventos (deportes, desfiles, conciertos, protestas), cine, fotografía y video aéreo, periodismo, investigación arqueológica y biológica, construcción e inspecciones de obras, delivery (envío de correo y comidas rápidas), manipuladores de materiales nocivos o peligros, logística, mantenimiento 
(líneas eléctricas, generadores, sistemas eólicos, maquinaria y otras estructuras), inspección de alcantarillado, minería, y por supuesto ocio.

Existen otros desarrollos y aplicaciones más específicos, como el emplear los drones como una flota de satélites, que funcionen con energía solar y actúen como un sistema de red de conexión de internet para zonas rurales. También, se plantea el uso de una flota de drones como sistema de distribución de vacunas y/o asistencia sanitaria en zonas apartadas. Una potencial aplicación de los drones, es en el diseño de los microUAV y nanodrones, cuyo tamaño sería equivalente al de un ave y un insecto respectivamente, ya existen prototipos militares, como el ZALA 421-08 y ZALA 42112 rusos, el Bird-Eye y el Skylite israelí, el Bayraktar turco, entre otros, cuya electrónica está basada en micro y nanotecnología, su finalidad, espionaje, rastreo, monitoreo y a futuro sistemas de ataque y defensa furtivos.

A nivel civil, los drones están siendo empleados para espionaje, ataque dirigido y como kamikaze por bandas criminales [1] y por grupos terroristas [2] como el Hizbulah, Daesh, AIQaeda e ISIS. Este actuar ha generado que muchos países hayan restringido el uso de drones en las ciudades y zonas críticas, en la que a la par, se han gestado propuestas de automatización de robots y otros dispositivos militares, incluidos por supuesto los drones, combinando estas tecnologías, con el fin de intersectar objetivos y atacar. Un ejemplo al respecto, es el dron supersónico UCAV X-47B [3], que se caracteriza porque despega y aterriza sin intervención humana, sumado a que dispone de armas de defensa y ataque de alto poder. Otros drones parecidos al anterior son el Mantis, el Reaper y el Taranis. [4] Con este tipo de desarrollos, se pretende a futuro crear los LAW (Lethal Autonomous Weapon) [5, 6], que en esencia son sistemas robóticos inteligentes con la autonomía de aniquilar, en la que rastrea al objetivo y/o selecciona anulándolo por los medios necesarios; lo que supone que se suprime de la ecuación la decisión humana y todo lo que ello acarrea en cuanto a aspectos éticos y morales [7]. En este sentido, se han manifestado en contra de este tipo de desarrollos diversos organismos gubernamentales y no gubernamentales, que aunque no se sabe que ya exista un dron o robot con estas características, si es cierto que con el desarrollo tecnológico actual y venidero, es muy probable que dentro de pocos años se escuche sobre estos sistemas y se vea en operación en algún lugar del mundo.

Con tanta diversificación de aplicaciones tecnológicas y necesidades a nivel de seguridad mundial, es solo cuestión de tiempo que el desarrollo de un sistema de drones cuyo fin sea el monitoreo permanente desde el aire de toda el área metropolitana de una ciudad sea una realidad dentro de poco tiempo, en la que convergen tecnologías como: la inteligencia artificial (IA) mediante implementaciones como el aprendizaje profundo y la visión artificial, los sistemas biométricos, la robótica y sistemas de control avanzados, el internet de las cosas, la energía híbrida, la comunicación móvil $4.5 G$ y $5 G$ que implican redes de alta velocidad, entre otros. Con estas tecnologías integradas en drones de altas prestaciones, el nivel de seguridad y el concepto propio de la misma van a cambiar, pues el apoyo a procesos como la detección y reacción ante eventos se va desarrollar in situ, dando a las fuerzas del orden un tiempo de reacción más rápido, minimizando con ello el impacto de la delincuencia en la sociedad y de todo acto que atente contra la vida o el medioambiente, incluyendo la atención ante emergencias, desastres, salud pública y movilidad, entre otros.

\section{Drones}

Los vehículos aéreos no tripulados (UAV, Unmanned Aerial Vehicles) o drones, son naves aéreas controladas de forma remota por personal en tierra, aunque existe ya en el mercado algunos UAV que cuentan con sistemas inteligentes [8] que les brinda cierta autonomía en su vuelo y desarrollo de diversas tareas, tales como la navegación y registro del entorno.

La tecnología en cuanto a sensórica, microelectrónica y nanoelectrónica, video y comunicaciones, han madurado lo suficiente como para dar el siguiente paso en aplicaciones de drones al sector civil a gran escala. Tanto así, que el incorporar la robótica, la IA [9] y visión artificial a los drones es un hecho, en la que se brinda la capacidad de desarrollar tareas en forma autónoma, minimizando y/o eliminando la necesidad de asistencia humana directa. Esto se puede hacer, porque la aviónica de estos vehículos es relativamente sencilla, debido a los múltiples dispositivos integrados acoplados a sistemas guía inerciales (acelerómetros [10, 11] y girómetros [12]), altímetros [13], controles de vuelo y GPS, entre otros dispositivos. Por ejemplo, dentro de las aplicaciones habituales de un dron de última generación, está el de disponer de un software inteligente que permite su manipulación gestual, al igual que poseer un sistema anticolisión y electrónica avanzada para geoposicionamiento, tomando como referente los sistemas GPS y el GLONASS (Global'naya Navigatsionnaya Sputnikovaya Sistema, Sistema Global de Navegación por Satélite).

Los drones son una herramienta tecnológica cuyo radio de aplicación se expande cada día a diversos campos tal como se ha anotado. Por ende, el llevarlo al nivel de seguridad a gran escala en una ciudad, abre todo un nicho de mercado de desarrollo y aplicaciones sin precedentes, a parte del potencial uso en el campo militar. Esto conlleva un cambio de paradigma a la denominada tecno-sociedad, en la que este tipo de iniciativa se va a implementar de manera generalizada en varios países antes del 2020 , debido al creciente nivel de terrorismo y delincuencia organizada, entre otros factores que alteran el orden público en las grandes ciudades. El establecer la forma cómo se va a monitorear y velar por la seguridad de la sociedad, va a ser cedido en parte a las máquinas, y aunque detrás de ellas esté el hombre, es solo cuestión de tiempo donde 
la autonomía se relegue no solo a los drones sino a otros dispositivos robóticos y máquinas inteligentes; los cuales estarán acoplados en línea con la red de sensores insertos o colocados en las vías públicas, en puentes, edificios y hogares (sistemas inmóticos y domóticos) [14], en sistemas de transporte público y privado, en máquinas y electrodomésticos, cuya particularidad es que estos estarán conectados en red permanentemente, lo que dará otro significado a lo que actualmente se define como internet de las cosas [15]. Cabe mencionar, que se estima que para el 2020 van a existir más de 50 mil millones de dispositivos conectados (omnipresentes), es decir, más que la población mundial estimada para esta fecha (7.5 mil millones). [16, 17] Por consiguiente, el presuponer la conectividad, monitoreo e intercambio de datos de los sensores dispersos por toda la ciudad versus drones y otros sistemas electrónicos, no es una utopía, a la que se suma una gran cantidad de sistemas de seguridad, empezando por las cámaras de video y demás sensores dispuestos por toda la metrópoli. Para que esto ocurra, antes se debe estructurar el factor de seguridad, pues actualmente presentan ciertas vulnerabilidades en materia de integridad, confidencialidad y disponibilidad de la información que delincuentes informáticos pueden aprovechar, por lo que se espera esté solucionado en los próximos años.

\subsection{Características de la flota de drones}

Los drones civiles presentan una autonomía de vuelo limitada, que varía de pocos minutos a varias horas (4 horas aproximadamente en el momento de escribir este artículo), sumado al peso o carga adicional. Por consiguiente, las aplicaciones son locales y restringido al radio de alcance del sistema de radiocontrol, que está condicionado a un espacio relativamente despejado. Esta limitante, supone seis problemas que están en proceso de solución, conforme al estudio realizado, si se piensa en una flota de drones revoloteando por toda la ciudad, que es el eje central de investigación del proyecto denominado "Sistema inteligente de monitoreo aéreo de seguridad metropolitana" o SIMASM. Los puntos de estudio son:

- Autonomía de vuelo: entendida en el contexto de la cantidad de energía que debe disponer las baterías para la ida y vuelta del dron de manera segura. Esto supone que el tiempo de vuelo está supeditado a la duración de las baterías.

- Disponibilidad de carga: se entiende la capacidad de carga útil o peso que puede transportar el dron en y durante el vuelo.

- Comunicación: la calidad de la transmisión y recepción de información entre el dron y una estación base para transmitir información en tiempo real, al igual que la interacción con otros sistemas de comunicación satelital y otros dispositivos en tierra, incluso con otros drones. Este sistema requiere de un mayor ancho de banda para garantizar una comunicación y transmisión de datos efectivo, sumado al factor seguridad de datos codificados.

- Suministro de energía: normalmente los sistemas de carga eléctrica de los drones son desde una toma de corriente con un adaptador a un voltaje determinado según la zona continental. Lo que implica que no hay un sistema de carga ideal que permita al dron reabastecer y reanudar su labor de manera rápida y autónoma.

- Programación inteligente: desarrollo e implementación de software y hardware basado en IA y robótica, que permita a los drones realizar el registro autónomo de datos y en ciertas circunstancias la toma de decisiones.

- Regulación: El uso de los drones en la actualidad presenta problemas a nivel normativo y jurídico [18, 19]. Aunque algunos países han tomado cartas sobre el asunto, aún falta establecer aspectos legales en cuanto a certificación, uso del espectro radioeléctrico y planes de integración en el espacio aéreo [20]. En este último aspecto, actualmente se prohíbe el uso de drones por personal civil en núcleos urbanos, sea de día 0 de noche, al igual que sobre concentraciones de personas, sea en entornos abiertos o cerrados.

La diferencia que hay entre el dron civil y militar, se fundamenta en los problemas citados, aunque existe otros, pero para las particularidades del proyecto en mención son las prioritarias. Para el caso de los drones militares, estos pueden ser tácticos, operacionales y estratégicos, que se caracterizan por tener una mayor autonomía de vuelo, recorrer grandes distancias y portar mayor peso (por ejemplo, sistemas de monitoreo, detección y armas defensivas como ofensivas), a lo que se suma su envergadura y potentes motores. Además, el tipo de material compuesto con que están construidos les permite alivianar su peso, repercutiendo en el aumento de carga útil y el menor uso de energía o combustible en su trayecto. Cabe mencionar, que la inversión en el desarrollo militar de drones se ha venido incrementado cada año, no solo en las superpotencias sino en la mayoría de países de todo el mundo y Latinoamérica no es la excepción, debido a que la inversión en este tipo de tecnologías es viable en cuanto a I+D+i que con tecnologías armamentistas robustas.

Existe una excepción a los drones citados anteriormente, cuya aplicación es en servicio público, como el taxi aéreo autónomo en la ciudad de Dubai denominado Volocopter $[21,22]$. Este es un vehículo tripulado de altas prestaciones, híbrido entre un dron y un helicóptero (multicóptero), con diversas variantes, monoplaza (VC100), biplaza (VC200-D-MYVC), el de cuadriplaza (VC 400) y sin tripulación (UVC100, 200 y 400). El VC200 entró en producción desde el año 2016, y aunque existen otros prototipos, este multicóptero está homologado por las autoridades aéreas y es un hecho en términos comerciales, por lo que en los próximos años se implementará en otras ciudades del mundo, abriendo un nuevo mercado a los vehículos aéreos tripulados y no tripulados. 


\subsection{Oportunidades detectadas}

Basado en el hecho que las sociedades cada vez más están concentradas en las ciudades, la seguridad de estas se pone a prueba día a día, máxime cuando está aumentando progresivamente el índice delincuencial organizado, las mafias, el terrorismo y la migración a gran escala, en la que actualmente se han convertido en factores comunes a nivel mundial, con contadas excepciones. Adicional a este panorama, las inestabilidades políticas de ciertas regiones y los problemas medioambientales globales, advierten que el nivel de inseguridad y desorden público va en aumento para las grandes urbes en los próximos años.

Previendo este cuadro poco alentador, las tecnologías integrada a los drones, como la citadas anteriormente, abren un sinnúmero de oportunidades de desarrollo técnico y tecnológico sin precedentes, en la que se propende dar solución a la problemática planteada. Por ejemplo, ya se puede reducir la interacción entre el hombre y la máquina, bajo la premisa de la integración de la programación no lineal (redes neuronales, algoritmos genéticos, lógica difusa, etc., que convergen al aprendizaje profundo y aprendizaje máquina), por tanto, cuando se habla de autonomía de una máquina, el concepto no debe tomarse tan a la ligera. Los sistemas de autodiagnóstico basado en sensores de monitoreo y electrónica flexible ya están al alcance de la industria civil, lo que reduce los niveles de inspección humana en una máquina, pues ésta emite un autodiagnóstico, en la cual por ahora, el humano toma la decisión en última instancia del mantenimiento o no, pero a futuro será la máquina quien decida, incluso para auto-repararse. De igual manera, los desarrollos en software inteligente están haciendo lo suyo en cuanto al autodiagnóstico y reparación, cuya implementación está a la vuelta de la esquina. Ejemplos de ello está el sistema HP SureStart [23] y el ClearView [24].

Continuando con la propuesta, una flota de drones debidamente programados y coordinados como el SIMASM, podrá actuar como apoyo a las fuerzas policiales en una ciudad, estableciendo una red de operación completa (policía, bomberos, emergencias y cuerpos de rescate). Con ello, se reducen los riesgos en comprometer vidas humanas en operaciones críticas, bien sea de rescate, asistencia clínica y de atención ante incidentes de orden público, entre otros. Como el espacio aéreo en el área metropolita no está ocupado por otros dispositivos, conforme a lo dispuesto por la ley, el nivel de operaciones de una flota de drones se puede desarrollar sin inconveniente alguno. Además, las comunicaciones por radiofrecuencia y móviles están lo suficientemente desarrolladas, como para poder integrarse con la tecnología de los drones y establecer una red de comunicación fiable y segura en toda el área y perímetro de una ciudad.

Otra oportunidad detectada, es que la industria de los drones es variada y dinámica, por lo cual, el costo relacionado con su inversión es asequible, al igual que la incorporación de nuevos desarrollos técnicos y tecnológicos sobre los mismos, propendiendo de esta manera a que su implementación sea relativamente corta, con un beneficio incalculable en materia de seguridad, dando paso al soporte del concepto de Ciudad inteligente (Smart City), porque a esta flota de drones se puede integrar otras tecnologías para el monitoreo y diagnóstico de la ciudad, bien en cuanto a índices de polución y/o contaminación ambiental, análisis atmosférico y radiancia (para uso en sistemas eólicos, solares e híbridos), inspección de anomalías estructurales tanto de vías como de construcciones, aspectos clave para minimizar riesgos- y finalmente planificación urbana, entre muchas más aplicaciones.

\section{Comunicación entre drones}

Los costos de desarrollo de un dron fluctúan según las tecnologías integradas, en la que se puede ampliar su rango de herramientas según las funciones para las que se desee implementar. Por ejemplo, cámaras de visión nocturna, sistemas telemétricos, sensores infrarrojos y de ultrasonido, radares, sensores laser de largo alcance, al igual que sistemas de comunicación encriptados, son algunas tecnologías disponibles en el mercado que se pueden integrar a un dron, al cual por supuesto se debe hacer reingeniería para adaptar estos dispositivos en cuanto a su tamaño, funcionalidad y operatividad. Todas estas tecnologías eventualmente se van a ir incorporando a un sistema autónomo, gestionado por software y hardware inteligentes, que le permita al dron realizar misiones de monitoreo de un área específica metropolitana de manera automática, en la que incluso se tome decisiones bajo el supuesto de la incorporación del aprendizaje profundo.

En cuanto a la programación de los drones, los desarrollos de código para IA y VA pueden ser realizados en diferentes plataformas de programación como Python, Haskel, Matlab, OpenCV, java, Deepcoder, entre otros, que pueden ser combinados con el software propio de las cámaras, sensores, actuadores y aviónica del dron para que realice la captura de video e imágenes, monitoreo, rastreo, detección y comunicación en vuelo [25] sobre un objetivo en particular, sumado a la intercomunicación con otros drones y las estaciones base. Esto implica, que el siguiente paso para implementar drones a gran escala con fines específicos de seguridad, va a ser una red coordinada de estos dispositivos que circunden el área metropolitana. Por consiguiente, cualquier aplicación de software de la nave va a recibir y/o transmitir datos, interpretando $y / 0$ ejecutando las instrucciones necesarias para un monitoreo coordinado entre otros drones en vuelo y, las autoridades en tierra y el aire, por ello se requiere de unas etapas de planificación operativa de estas máquinas.

En términos generales el método de planificación de vuelo incluye las siguientes etapas para el control de un dron: [26]

- Verificar el estado del dron en cuanto a funcionalidad y comunicación. 
- Conectar con el controlador de vuelo.

- Obtener flujo de datos de vuelo del controlador.

- Adquirir flujo de señal y comunicaciones.

- Verificar estado de seguridad del vuelo autónomo.

- Determinar de manera autónoma el movimiento de la aeronave.

- Enviar comandos de vuelo al controlador y registrar el vuelo.

Una vez desarrollas estas etapas, los sistemas basados en IA, VA y máquinas de aprendizaje [27], se encargarán de activar tanto sensores como actuadores robóticos, para que inicie el escaneo de un perímetro, sumado a la comunicación con otros drones que están en el mismo estado de vuelo y el centro de comando central ubicado en tierra. En cuanto a los actuadores, estos se emplearían para rotación de cámaras terrestres o aéreas, al igual que para activar los diversos sensores de monitoreo, detección y rastreo. Todo esto se realizará mediante comunicación móvil - dron- central y central dispositivos móviles de las autoridades y CCTV vía WiFi $u$ otras redes de comunicación. Tal como se indica en la figura 1.

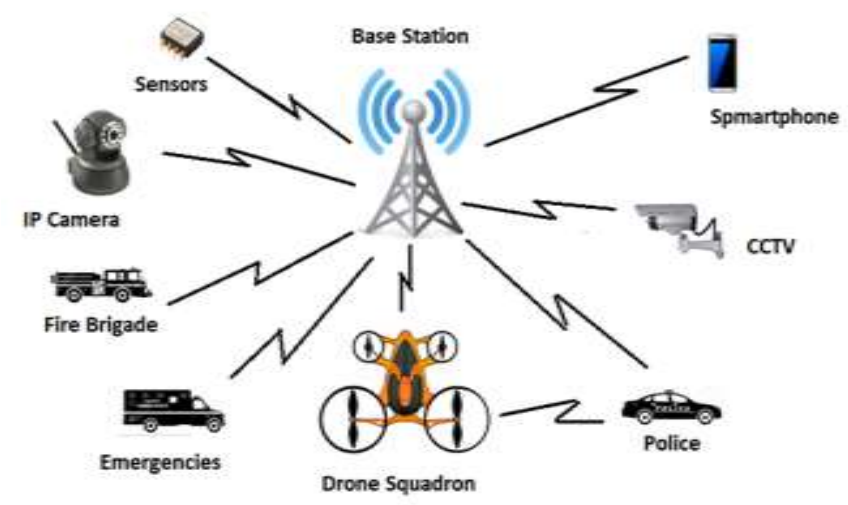

Figura 1. Esquema de la comunicación móvil - droncentral y central - dispositivos móviles de las autoridades y CCTV vía WiFi u otras redes de comunicación

La disposición de los drones en escuadrones en un entorno metropolitano, aprovecha la telefonía móvil 4G+ y $5 \mathrm{G}$, usando los canales GPRS para enviar de información por un canal dedicado a una estación base, quien se encarga de actuar como puente de comunicación con los demás sistemas de comunicación tales como: cámaras de circuito cerrado dispersas por toda la ciudad, cámaras IP y dispositivos móviles que dispone la policía y comercio, etc. Además, los drones coordinaran su desplazamiento, monitoreo y recarga de sus baterías entre ellos y la central, con lo cual nunca va a quedar un sector de la ciudad desprotegido, pues habrá otro dron de respaldo. Fuente. Elaboración propia.

Con el avance progresivo de las cámaras de video [28], se convierten literalmente en los ojos de las fuerzas policiales en el aire, en la que permite efectuar la medición de variables como aceleración y velocidad del dron respecto a la superficie de navegación, por lo cual, el registro de un evento estático o en movimiento es extrapolable.

En cuanto a la comunicación, la implementación en una flota de drones no es compleja, pues se tiene la infraestructura de las redes de comunicación de la propia policía o por medio de un canal dedicado móvil empleando el protocolo GPRS, que funciona sobre redes GSM y UMTS, en la que se debe hacer un desarrollo con microcontroladores (en un sistema embebido), que gestione la comunicación del GPRS tales como el sistema Wismo 228 [29] o el L95, según experiencias previas de laboratorio. Como complemento a lo citado, debido a la escalabilidad de información que va a tener el sistema propuesto, es importante pensar en implementar la computación de alto rendimiento [30] con el fin de maximizar la eficiencia, tolerancia a fallos, robustez y fiabilidad en el flujo y transferencia de datos. La idea de este desarrollo, es que la forma de operación del dron está restringida a un área específica y de manera coordinada, considerándose como un cluster robótico [31] así:

- La altura estándar que puede alcanzar un dron de última generación es de 500 a 2500 m, permitida en algunos países en zonas despobladas, pues puede alcanzar mayor altitud, por ejemplo, el Phantom 4 alcanza $6000 \mathrm{~m}$. Para el caso del proyecto en cuestión, la altura estimada es del orden de $900 \mathrm{~m}$ a $1.5 \mathrm{~km}$, pues el pilotaje es autónomo o semiautónomo, ya que la operación se gestiona desde una central de operaciones fija o móvil. Este aspecto de la altura es fundamental porque el radio de cobertura de las cámaras y sensores está limitado conforme asciende el dron.

- A mayor altura, la ventaja de comunicación entre los drones aumenta al estar estos en línea vista. Este es un elemento importante, puesto que, al efectuar la transmisión de información entre drones para coordinar zonas de cubrimiento, recepción, escaneo y monitoreo de un evento, se requiere que el espacio entre estos esté libre de obstáculos, tales como edificaciones.

- El área de cubrimiento de un dron está restringido a la autonomía de sus baterías. Como referente para un dron estándar, el alcance es de 500 a $600 \mathrm{~m}$, que comprende el radio visual del piloto. Calculando un tiempo de duración de 4 horas como máximo, a velocidades que fluctúan entre los 60 a los $130 \mathrm{~km} / \mathrm{h}$, el alcance estándar máximo se estima que es de aproximadamente entre 120 y $260 \mathrm{~km}$, que es una distancia suficiente para cubrir una gran ciudad. La velocidad lo es todo en el caso de una persecución y/o rastreo de un objetivo. Por ejemplo, actualmente el dron civil más rápido es el RacerX [32], con una velocidad aproximada de $290 \mathrm{~km} / \mathrm{h}$, lo que da a entender que la velocidad no es una limitante en materia de desarrollo e implementación de un escuadrón de drones. 
Pruebas como las realizadas en el Reino Unido por la policía, cuenta con escuadrones de drones [20] que trabajan las 24 horas del día, permiten establecer el rango de cobertura y asistencia para el rescate y vigilancia, que es limitado por el momento. Para el caso propuesto del SIMASM, el escuadrón de drones estará conectado al sistema de videocámaras y sensores dispuestos en puntos estratégicos en toda la ciudad, en la que eventualmente se evidencien problemas de orden público, accidentes, vías de alto flujo vehicular y potenciales fallas estructurales 0 naturales. Esta información se complementaría con las propias cámaras y sensores de la flota de drones, que contarían con la tecnología que permiten operar software para el análisis de información digital y multimedia, al igual que programas basados en aprendizaje profundo, para efectuar el análisis sobre sistemas de reconocimiento acústico y reconocimiento visual, que permita identificar al dron señales de auxilio o ataque (por ejemplo armas de fuego accionadas o señal de auxilio por parte de una víctima) mediante filtros supresores de ruido, a través del uso de algoritmos heurísticos y/o similares. Cabe mencionar, que en el mercado policial ya existe el dispositivo que permite localizar el origen de un disparo antes que la bala llegue a su destino. [33] La limitante es el tamaño, sobre la cual ya se está trabajando para hacerlo portátil. Otro dispositivo que actualmente pertenece al campo militar, son los sistemas de radar que permiten el registro de personas a través de cualquier obstáculo, el cual en el campo civil será muy útil, en particular para buscar víctimas de siniestros 0 accidentes.

\section{Autonomía y recarga}

La autonomía de un dron está en proporción directa a la carga que soporta, sumado al área de cobertura, cuyo tiempo oscila entre unos pocos minutos a varias horas [34]. Esta limitante de vuelo se soluciona al implementar sistemas de recarga en puntos fijos y altos, dispuestos a lo largo y ancho de la ciudad estratégicamente ubicados, tales como, las azoteas de edificios o torres diseñadas para tal fin, donde los drones después de hacer su ronda, van a estos puntos, recargan y entre tanto otros drones los reemplazan en su área de trabajo, a lo que se denomina como turnos cíclicos.

Existe una propuesta desarrollada por la empresa Skysense, [35] que ha ideado una solución portátil y solar para la recarga de la batería de un dron a través de una almohadilla de carga inalámbrica, conocida con el nombre de Droneport. Su funcionamiento es muy sencillo, el dron aterriza sobre la almohadilla que se activa al contacto e inicia la recarga automáticamente por señales de radiofrecuencia y a la par se puede transferir datos in situ. - La tecnología Droneport tiene su equivalente en el mercado para la recarga de dispositivos móviles denominado "Charging Pad for Mobile Phones" que emplea señales inalámbricas.- Lo que implica que se puede extender estas tecnologías a otros drones más grandes y compactos, con la particularidad que el sistema cargador no emplearía la toma de corriente o baterías portátiles, sino que va adquirirla a través de un sistema híbrido (eólico y solar) $[36,37]$, por lo que en términos ecológicos es viable y económico de implementar (Ver figura 2), yendo en armonía con el concepto de ecotecnología.

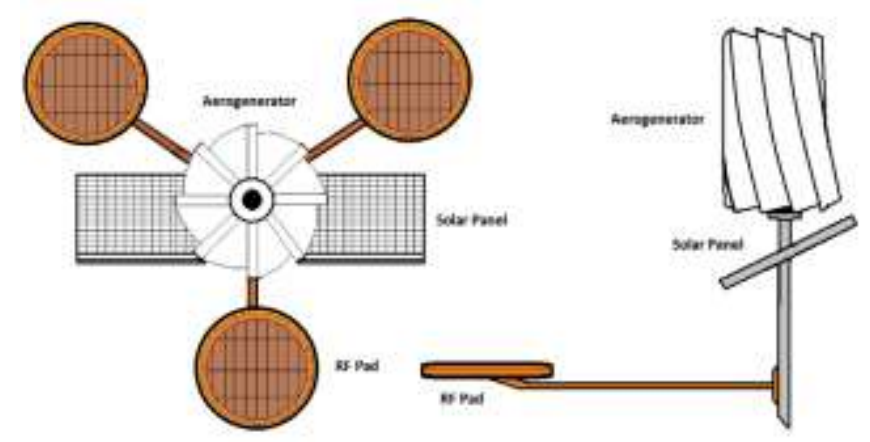

Figura 2. Sistema híbrido de carga autónoma inalámbrica de energía eólica y solar para una flota de drones (patente en proceso).

Los drones aterrizan sobre la plataforma que consta de una almohadilla (circunferencias en la gráfica, RF Pad) que recarga la batería del dron por medio de señales de RF. La plataforma tiene espacio para una flota de tres drones. El espacio se puede optimizar si se incorpora un sistema hibrido tipo wind screw, que incorpora en un solo dispositivo paneles solares y un aerogenerador urbano vertical. Con ello, se obtiene energía limpia para el sistema y para usos alternos como iluminación y alimentación para los sistemas de comunicación de la plataforma y alrededores. Fuente. Elaboración propia.

La energía que se obtiene de un sistema híbrido redundante para el sistema propuesto, cumple con los estándares de estabilidad y obtención energética, con bajo impacto en el medioambiente y niveles de ruido aceptables según estándares internacionales. Con la energía que se obtiene de este sistema híbrido, es suficiente para la recarga de los drones y alimentar los sistemas de comunicación en tierra, sumado a que es independiente de la red eléctrica, por lo que no hay costos por este concepto, de hecho, el sistema puede suministrar energía eléctrica a las luces de alerta nocturna y alumbrado público aledaño. El sistema híbrido de carga autónoma inalámbrica de energía eólica y solar para la flota de drones, es una opción ideal en la que estos van a operar las 24 horas del día, 7 días de la semana, todo el año, si así se dispone.

Otro elemento a explorar, es la mejora en la autonomía de los drones en cuanto al alcance de la señal, que mediante amplificadores se puede expandir a un rango de varios kilómetros, los cuales ya existen en el mercado, de hecho, se puede emplear el espectro de radiofrecuencia que emplea la policía para ser usado como canal de comunicación o un canal de telefonía móvil dedicado.

Para el monitoreo con un escuadrón de drones como el SIMASM, el rango estimado varía según el área de 
cobertura dentro y en la periferia de una ciudad. Como caso particular para una ciudad como Bogotá D.C, su área urbana total es de $528 \mathrm{Km}^{2}$, con aproximadamente ocho millones de habitantes. [38] Con un cálculo geométrico sencillo, se estima que el área de cobertura por dron es de aproximadamente $12.6 \mathrm{~km}^{2}$ (área de circunferencia con radio de $2 \mathrm{~km}$, con una altura de referencia de $500 \mathrm{~m}$ ), por lo cual se requiere un total de 42 drones. Si se amplía la escala de cobertura a un radio de $2.5 \mathrm{Km}$, el número de drones se reduce a 27 . Ahora, se debe tener en cuenta que no toda la ciudad es insegura y que algunos sectores no requerirán de vigilancia permanente, por lo cual el número de drones se reduciría a menos de la mitad. Pero como la operatividad de los drones no es permanente, y requiere de reemplazo, sumado al uso permanente, el número total debe mantenerse duplicado o triplicado según las necesidades, para mantener la rotación y no dejar desprotegido en ningún momento alguna zona crítica de la ciudad. En cuanto a la autonomía y eficiencia están dictadas por la programación algorítmica inteligente, por lo que el nivel de aprendizaje de los drones, estará literalmente en proporción exponencial, sumada al constante crecimiento de los procesadores, por lo cual las tarjetas de circuito integrado pueden ser reemplazadas y/o actualizadas cuando sea necesario.

Tal como se ha descrito la propuesta es un sistema multiplataforma escalable, por lo que los costos de actualización serían bajos, con un beneficio incalculable para una ciudad. La seguridad en un entorno urbano es una necesidad que requiere de soluciones prontas, con costos que no sean prohibitivos, por lo cual el proyecto en mención es una opción viable a corto y mediano plazo, en la que conforme el sistema se implementa o se haya implementado, se pueden ir integrando otras tecnologías, manteniendo el sistema actualizado y activo con una inversión razonable para la ciudad.

\section{Discusión}

La propuesta de emplear drones en una ciudad es relativamente reciente, la cual ha venido tomando fuerza en diversas ciudades del mundo, con base en los ataques terroristas empleando drones caseros $\mathrm{y} / \mathrm{o}$ estándar modificados para portar cargas explosivas [39], lanza cohetes e incluso artillados. La problemática sobre la autonomía de vuelo de los drones, prácticamente ha sido subsanada. El paso a seguir está dirigido hacia la coordinación de vuelo y sistemas de seguimiento y rastreo de objetivos, que puedan ser centralizados y a la vez aislado para ciertas funciones, garantizando de esta manera que todo el sistema no sea vulnerable a un ataque informático que comprometa toda la infraestructura de comunicaciones y flujo de datos de la flota de drones, y por ende, exponga a las zonas sensibles de una ciudad a un ataque coordinado empleando la misma flota.

Es claro que se requiere soluciones a corto plazo para reducir la problemática de seguridad local y global, donde el terrorismo tiene agobiado a las grandes ciudades, secundado por la delincuencia común y organizada, que se han venido incrementando por los diversos conflictos políticos internos y externos presentes en algunos países en diversas regiones inestables del mundo. A lo anterior se suma, que el monitoreo de las vías en una ciudad, la atención a emergencias, e incluso siniestros, no podrán ser atendidos y suplidos a tiempo por unidades terrestres, tampoco por helicópteros, ya que estos no pueden aterrizar en zonas poco amplias o pobladas, o en el peor de los escenarios, zonas devastadas. Basado en este panorama, es innegable que los drones pueden ser una gran herramienta funcional y operativa al interior de una ciudad, pues permiten cubrir una mayor área en un menor tiempo, lo cual es una variable clave para atender cualquier emergencia que se presente.

Con la tecnología actual implementada a los drones, la información capturada se puede procesar en mapas e imágenes tridimensionales de cualquier punto de la ciudad, al igual que de personas y vehículos. Con un sistema como el SIMASM, la reducción de tiempo de respuesta es literalmente inmediata, sumado a que el personal encargado del sistema es reducido. Aunque la propuesta es similar a la desarrollada por la policía británica, difiere en la forma cómo la flota de drones va operar y recargar en sus rondas. Cabe mencionar, que recientemente se ha anunciado por parte de DARPA (Defense Advanved Research Projects Agency), implementar una red de drones para monitorizar la actividad de cualquier aeronave de tamaño reducido que vuele en el espacio aéreo urbano y militar. [40] Este escuadrón de drones difiere en algunos aspectos del proyecto propuesto en cuanto a su propósito, pues lo que busca es derribar cualquier dron u objeto que vuele sin autorización por determinadas zonas de la ciudad. Esto supone que lo expuesto en el presente artículo converge a las diversas iniciativas como la británica, americana o coreana, donde esta última trabaja en implementar en un futuro no muy lejano flotas de drones para la seguridad urbana. Por ejemplo, para el caso de Corea del Sur, anunció en el 2016 una inversión que superaba los 1000 millones de dólares en el sector de desarrollo de drones avanzados para literalmente cualquier tipo de industria. Este actuar da a entender el interés e importancia de las naciones de protegerse, creando de paso nuevas fuentes de trabajo, en la que se empleen tecnologías emergentes relativamente baratas, que representen un alto grado de fiabilidad, máxime cuando la delincuencia organizada y grupos terroristas ya disponen de ello para sus actos ilícitos.

\section{Conclusiones}

Los drones presentan un potencial de uso en el área de monitoreo y seguridad en entornos civiles, al igual que la ventaja en cuanto a su rapidez y desplazamiento sobre una zona urbana y periferia, superando fácilmente cualquier obstáculo, ofreciendo servicios de video, imágenes e información en tiempo real sobre cualquier evento que se presente en su área de cobertura. Con un escuadrón de drones interconectados entre sí, se crea toda una infraestructura redundante de seguridad, que 
trabaja en conjunto con tecnologías existentes como los sistemas de cámara de circuito cerrado, telefonía móvil y redes inalámbricas para comunicación dedicada a través de la misma. Estos enjambres de vehículos aéreos no tripulados pueden desplegarse para realizar tareas tales como: búsqueda y atención de personas ante cualquier tipo de desastre natural, emergencias, accidentes 0 ataques terroristas, búsqueda y rastreo de sospechosos, sumado a la inspección y vigilancia en general de una ciudad, en particular zonas críticas, tales como: acueductos, hospitales, centros de suministro energía, infraestructura gubernamental y/o militar, entre otros.

Se espera que, con el uso de los drones en un ambiente urbano, lo daños colaterales a las fuerzas del orden versus delincuencia y/o terrorismo sea menor, pues las reacciones ante un evento de agresión o conflicto, son variables y dinámicas en el ser humano, aspectos que en estos sistemas no va a existir a futuro. Cabe mencionar que las discriminaciones acerca de la intención de actuar $o$ no ante un evento quedan supeditados a las fuerzas del orden, aunque el sistema podría eventualmente hacerlo, conforme va aprendiendo de los expertos y de su propia experiencia. En este sentido, surgen preguntas como ¿si algo falla en un procedimiento a quien se culpa al humano o la máquina? ¿Las máquinas pueden quedar ausentes de toda responsabilidad civil? Estas preguntas y otras que surgirán son inevitables, pues se advierte un cambio de paradigma en la tecno-sociedad, donde para las generaciones actuales quizás sea contraproducente esta propuesta, pero analizándola desde la óptica de la seguridad global, sea aceptable, garantizando con ello una mejor calidad de vida a las generaciones venideras. La propuesta implícitamente presenta aspectos controversiales a nivel social, cultural y político, pues no es ningún misterio, que al plantear un sistema que monitoree una comunidad permanentemente, van a existir detractores que van a ver vulnerado de alguna manera su derecho a la privacidad. Aunque no se tocó este asunto, no puede dejarse de lado y enfatizar que cualquier cambio tecnológico a gran escala genera controversia, máxime cuando su aplicación afecta directa o indirectamente a la comunidad en general, a pesar que su fin sea de carácter altruista.

A futuro los sistemas robóticos de seguridad y por extensión los drones, tendrán acoplados sensores capaces de detectar marcadores biológicos de un objetivo, bien por el sudor del mismo o por patrones biométricos (huellas dactilares, reconocimiento de rostro, retina, iris, palma, forma de desplazamiento, etc.) [41, 42], al igual que permitirá escanear el cuerpo de un individuo o vehículo en busca de algún tipo de artefacto explosivo o armas. Toda esta información a posteriori estará concentrada en servidores, lo que supone la incorporación a esta flota de drones sistemas de cómputo de altas prestaciones que va a facilitar el monitoreo y seguimiento de objetivos, el desplazamiento y formación de vuelo coordinados, y flujo constante de datos entre las partes.
Desde el punto de vista industrial, una vez resuelto el problema normativo, las potencialidades del sector de los drones en el marco civil son amplios, tanto desde la óptica de la innovación o el desarrollo industrial, como de la generación de empleo cualificado. [20] Para finalizar, el desarrollo del sistema propuesto es relativamente rápido cuyo retorno de la inversión es de pocos años, con un impacto social sin precedentes, en la que se espera mejore el nivel de seguridad en las grandes ciudades de todo el mundo. Aunque lo expuesto pueda parecer ficción, la realidad es otra, la implementación a gran escala de flotas de drones en sectores de ciudades se está gestando, solo es cuestión de tiempo que se aumente su rango de acción, basado en un modelo igual o equivalente al expuesto en el presente artículo.

\section{Trabajos futuros}

Se pretende a mediano plazo desarrollar el sistema híbrido de carga autónoma inalámbrica de energía eólica y solar, una vez sea aprobada la patente. La propuesta como tal es fundamental para garantizar la carga de los drones in situ, aumentando de esta manera la autonomía de carga y recarga de estos en la ciudad, o en el lugar que se requiera.

Desarrollar un sistema embebido más robusto, que garantice la comunicación entre drones en pleno vuelo, y la posterior coordinación en cuanto a cubrimiento perimetral.

Adaptar los sistemas de carga de los drones a emplear en el proyecto por sistemas RF, que se ajusten a los requerimientos del sistema híbrido de carga autónoma inalámbrica de energía eólica y solar.

Investigar más a fondo sobre las características de transferencia de información de un dron versus la nube (modelo cliente-servidor), empleando sistemas de seguridad apropiados, que garanticen que los datos van estar protegidos, máxime para un sistema que va a estar expuesto a un medio tan dinámico. En este sentido se pretende estudiar la infraestructura de comunicación del sistema de drones y estación base, en la que se explore los modos de operación, ancho de banda y transferencia de información, entre otros.

Profundizar en el uso de los algoritmos de IA (Deep Learning) y visión artificial, con el fin de desarrollar software más robusto conforme a las condiciones de registro de información en vuelo, bien por las cámaras o por diversos sensores, que garanticen el análisis de variables más apropiado en un corto tiempo.

Diseñar o en su defecto aplicar reingeniería al tipo de dron ideal que se ajuste a los requerimientos expuestos en el proyecto, tales como autonomía, relación carga útil y peso total, entre otros, por lo que se requiere un estudio pormenorizado acerca de los modelos matemáticos [43, 44] existentes para revaluarlos dado el caso, con miras a optimizar la mecánica y dinámica del vuelo.

8. Agradecimientos 
El autor reconoce la contribución académico investigativa que la Universidad Estatal de Ohio brindó a quienes participaron en el curso TechniCity, propendiendo al desarrollo de una idea hasta llevarla a una propuesta viable, enmarcada en el contexto de mejorar la calidad de vida de las sociedades concentradas en las grandes ciudades, cuya tendencia para el presente siglo es la interconectividad, el medioambiente, la seguridad, la ubicuidad e inmersión, entre otros, los cuales forman parte de lo que se ha venido a denominar como ciudad inteligente o Smart City.

\section{Referencias}

[1] J. Nicas. El uso de drones por parte de criminales y terroristas preocupa a las autoridades. Jan, 29 2015. The Wall Street Journal. [Online]. Disponible en: https://www.wsj.com/articles/el-uso-de-drones-porparte-de-criminales-y-terroristas-preocupa-a-las-autoridades1422573625

[2] P. Cervera, Los drones del ISIS: cuando los juguetes voladores se hacen terroristas, September 5, 2017. [Online]. Disponible en: https://www.elconfidencial.com/tecnologia/2017-03-05/drones-daeshisis-terrorismo_1341002/

[3] P. A. García. "UCAVs: ¿Serán todos los futuros cazas aviones no tripulados?" Revista de Aeronáutica y astronáutica. Especial: 30 años del EF-18. No. 855, Julio-agosto 2016, pp. 601-607.

[4] M. Dobbing, A. Hailwood, C. Cole, Acknowledgements, Convenient killing. Armed drones and the 'playstation' mentality. For.org, Oxford, 2010. [Online]. Disponible en: https://dronewarsuk.files.wordpress.com/2010/09/convienient_killing1 .pdf

[5] M. Ekelhof, M. Struyk. Deadly decisions. 8 objections to killer robots. Pax, Netherlands, 2014.

[6] J. Foy. Autonomous weapons systems: taking the human out of international humanitarian law. Vol. 23, Dalhousie Journal of Legal Studies, 2014, pp 47-70.

[7] C. Mayer. Developing autonomous systems in an ethical manner. Cap 3. Autonomous systems. Issues for defence policymakers. Edited by: Williams A. P. Scharre P. D. Headquarters Supreme Allied Commander Transformation. NATO, OPTAN, Norflolk, United States, pp. 65- 82.

[8] S. Cristina. Radiografía de un dron: cerebro automático, sensores y una relación telepática con el piloto, 2014. [Online]. Disponible en: http://www.eldiario.es/hojaderouter/tecnologia/hardware/dronesRPAS-funcionamiento-inteligencia_artificial_0_328968201.html

[9] B. Raúl. E. Gerard. K. Samir. Inteligencia artificial avanzada, Universidad Oberta de Catalunya. Ediciones UOC, 2011.

[10] R. I Rincon-J, R. Ambrosio, J. Mireles. Análisis y caracterización de un acelerómetro capacitivo fabricado con tecnología polymump's, Superficies y Vacío 23 (3) 26-31, septiembre de 2010. [Online]. Disponible http://smcsyv.fis.cinvestav.mx/supyvac/23 3/SV2332610.pdf

[11] A. Baharodimehr, A. A Suratgar, A. and H. Sadeghi. Capacitive MEMS accelerometer wide range modeling using artificial neural network. J. appl. res. Technol. 2009, vol.7, n.2 [citado 2015-04-01], pp. 185-192. [Online]. Available: http://www.scielo.org.mx/scielo.php?script=sci_arttext\&pid=S1665$64232009000200006 \& \operatorname{lng}=\mathrm{es} \& \mathrm{nrm}=\mathrm{iso}$

[12] K. Schumacher, U. Wallrabe, J. Mohr. Wissenschaftliche Berichte. Forschungszentrum Karlsruhe Technik und Umwelt. FZKA 6361. Design, Herstellung und Charakterisierung eines mikromechanischen Gyrometers auf der Basis der LIGA-Technik, Institut für Mikrostrukturtechnik, Forschungszentrum Karlsruhe $\mathrm{GmbH}$, Karlsruhe, 1999.

[13] I. Rodríguez R. Sistema de Aterrizaje y Adquisición de Datos para un UAV, (Trabajo de grado) Instituto Politécnico Nacional. Escuela Superior de Ingeniería Mecánica y Eléctrica. México, septiembre 2007. [Online]. Disponible

en: http://tesis.ipn.mx/xmlui/bitstream/handle/123456789/10471/1434\%20 2007.pdf? sequence $=1$

[14] M. Matías, A. Gabilondo, V. Fernández, C. Ruiz, L. Falcone, F. Del villar F. y A. Milltino. Domótica e Inmótica - Instalaciones de telecomunicaciones para edificaciones. Ed. Alfaomega, 2016.
[15] R. Karen, S. Eldridge, L. Chapin. La internet de las cosas - Una breve reseña. Para entender mejor los problemas y desafíos de un mundo más conectado. Internet Society, 2015.

[16] D. Evans. Internet de las cosas. Cómo la próxima evolución de Internet lo cambia todo. Cisco. Informe técnico, 2011.

[17] J. Salazar y S. Silvestre. Internet de las cosas. Techpedia. České vysoké učení technické v Praze Fakulta elektrotechnická. 2016.

[18] K. Zickuhr and E. Stahl, Cities and Drones. What cities need to know about Unmanned Aerial Vehicles (UAVs), National League of Cities (NLC), Center for city solutions and applied research. 2016. [Online] Available: 12/NLC\%20Drone\%20Report.pd

[19] T. McDougal. Drones and the Law: The Sky's Not the Limit. 2016. [Online]. https://www.bhphotovideo.com/explora/video/tips-andsolutions/drones-and-law-skys-not-limit

[20] R. González, C. Herranz, A. Calvo. De los UAV a los RPAS. Revista Perfiles IDS, 2014. [Online]. Disponible en: http://www.infodefensa.com/wp-content/uploads/Af-Uavs-10-03.pdf

[21] Volocopter. Dubai beginnt 2017 weltweit ersten Testbetrieb autonomer Lufttaxis mit dem Volocopter, 2017. [Online]. Available: http://www.volocopter.com/

[22] A. Zosel. Volocopter. The "Green" helicopter of the future. Evolo. $2017 . \quad$ [Online]. Available: http://ecosummit.net/uploads/eco13_040613_1745_alexanderzosel_ev olo.pdf

[23] HP - Melissa. New HPSureStart: The Cure for BIOS Issues. September 17, 2013. [Online]. Available: http://h20435.www2.hp.com/t5/367Addison-Avenue-Blog/New-HPSureStart-The-Cure-for-BIOSIssues/ba-p/82999

[24] E. Naone, Software That Fixes Itself, A new tool aims to fix misbehaving programs without shutting them down. October 29, 2009. [Online].

Available: https://www.technologyreview.com/s/416036/software-that-fixesitself/

[25] J. M. Vergara and A. T. Andrés. Diseño e implementación del sistema de comunicaciones basado en CAN para la aviónica en un vehículo aéreo autónomo no tripulado. Rev. ing. univ. Medellin [online]. 2007, vol.6, n.11 [cited 2015-04-01], pp. 209-221. Disponible en http://www.scielo.org.co/scielo.php?script=sci_arttext\&pid=S1692$33242007000200014 \& \operatorname{lng}=$ en \&nrm=iso

[26] Universidad de Alicante. Sistema de control que permite el vuelo autónomo de drones, SGITT-OTRI. 2015, pp 3. [Online]. Disponible en: http://sgitt-otri.ua.es/es/empresa/documentos/ot-1502-drones.pdf

[27] Wireless Standard Modem. WISMO family, Sierra Wireless. Manual. [Online]. Available: http://docseurope.electrocomponents.com/webdocs/0eed/0900766b80eed43c.pdf

[28] F. Ofli, P. Meier, M. Imran, C. Castillo, D. Tuia, N. Rey, J. Briant, P. Millet, F. Reinhard, M. Parkan. Combining human computing and machine learning to make sense of big (aerial) data for disaster response. Big Data, 2016, 4, pp. 47-59.

[29] R. Näsi, E. Honkavaara, S. Tuominen, H. Saari, I. Pölönen, T. Hakala, N. Viljanen, J. Soukkamäki, I. Näkki, H. Ojanen. UAS based tree species identification using the novel FPI based hyperspectral cameras in visible, NIR and SWIR spectral ranges. Int. Arch. Photogramm. Remote Sens. Spatial Inform. Sci. 2016, XLI-B1, 1143-1148.

[30] L. Camargo, P Royo, X. Prats. On-Board High-Performance Computing For Multi-Robot Aerial Systems. Chapter 7 from the book Aerial Robots - Aerodynamics, Control and Applications. Intech Open Science- Open Minds. Downloaded from: http://www.intechopen.com/books/aerial-robots-aerodynamicscontroland-applications http://dx.doi.org/10.5772/intechopen.69443

[31] A. Marjovi, S. Choobdar, L. Marques. Robotic clusters: Multi-robot systems as computer clusters: A topological map merging demonstration. Robotics and Autonomous Systems. 2012; 60(9):11911204

[32] J. Condliffe. El dron más rápido del mundo bate el récord de velocidad a 290 kilómetros por hora, 2017. [Online]. Disponible en: https://www.technologyreview.es/s/8376/el-dron-mas-rapido-delmundo-bate-el-record-de-velocidad-290-kilometros-por-hora

[33] P. Cervera, Miras. sensores, drones y robots: la infantería del futuro ya está aquí. 2016. [Online]. Disponible en: https://www.elconfidencial.com/tecnologia/2016-03-07/las-armas-ytecnologias-que-usaran-los-soldados-del-futuro_1163875/

[34] D. A. González, G Rodríguez. Drones-An Open Access Journal Revista Journal drones. 2017, pp. 1-5. Doi:10.3390/drones1010001 
[35] I. Prates. Skysense Debuts Charging Pad for Parrot Bebop Drone at CES 2015 , 2015. [Online]. Available: http://mundogeo.com/en/blog/2015/01/05/skysense-debuts-chargingpad-for-parrot-bebop-drone-at-ces-2015/

[36] K. Bullis. TR10: Energías eólica y solar inteligentes. El 'big data' y la IA generan predicciones ultra precisas que permitirán aumentar la cuota de renovables en la red, 2014. [Online]. Disponible en: http://www.technologyreview.es/energia/45174/

[37] J. Martín. Energía solar fotovoltaica y energía eólica. AMV Ediciones, Madrid, 2014

[38] Censo 2005-2006. DANE. 2007. Marzo de 2017. [Online]. Disponible en: http://www.dane.gov.co/censo/files/presultados.pdf

[39] NY times. Pentagon Confronts a New Threat From ISIS: Exploding Drones, 2016.2 [Online]. Available: https://www.nytimes.com/2016/10/12/world/middleeast/iraq-dronesisis.html?smid=tw-nytimesworld\&smtyp=cur\&_r=1
[40] J. Montero. DARPA planea crear una red para monitorizar el tráfico urbano de drones. 2017. [Online]. Disponible en: http://www.todrone.com/darpa-planea-crear-red-monitorizar-traficourbano-drones/

[41] M. Tapiador, P. J. Sigüenza. Tecnologías biométricas aplicadas a la seguridad. Editor: RA-MA S.A. Editorial y Publicaciones, 2005.

[42] K. A. Gates. Our Biometric Future: Facial Recognition Technology and the Culture of Surveillance (Critical Cultural Communication). New York University Press, 2011.

[43] E Giraldo, P. Muñoz, J. Bonilla. Identificación y control de un Vehículo Aéreo no Tripulado tipo Quadcopter. Ing. USBMed, Vol. 7, No. 1, Enero-Julio 2016.

[44] S. Gutierrez. Modelado, simulación y control inteligente de UAVs. (Trabajo de grado). Escuela Técnica Superior de Ingeniería. Sevilla, septiembre de 2013 [Online]. Disponible en: http://bibing.us.es/proyectos/abreproy/60178/fichero/PFC_completo_S ara_Gutierrez_Bermejo.pdf 